\title{
High-order harmonic generation in graphene: Nonlinear coupling of intraband and interband transitions
}

\author{
Shunsuke A. Sato $\odot,{ }^{1,2, *}$ Hideki Hirori $\odot,{ }^{3}$ Yasuyuki Sanari, ${ }^{3}$ Yoshihiko Kanemitsu $\odot,{ }^{3}$ and Angel Rubio $\odot^{2,4, \dagger}$ \\ ${ }^{1}$ Center for Computational Sciences, University of Tsukuba, Tsukuba 305-8577, Japan \\ ${ }^{2}$ Max Planck Institute for the Structure and Dynamics of Matter, Luruper Chaussee 149, 22761 Hamburg, Germany \\ ${ }^{3}$ Institute for Chemical Research, Kyoto University, Uji, Kyoto 611-0011, Japan \\ ${ }^{4}$ Center for Computational Quantum Physics (CCQ), Flatiron Institute, 162 Fifth Avenue, New York, New York 10010, USA
}

(Received 13 October 2020; revised 4 December 2020; accepted 13 January 2021; published 29 January 2021)

\begin{abstract}
We investigate high-order harmonic generation (HHG) in graphene with a quantum master equation approach. The simulations reproduce the observed enhancement in HHG in graphene under elliptically polarized light [N. Yoshikawa et al., Science 356, 736 (2017)]. On the basis of a microscopic decomposition of the emitted high-order harmonics, we find that the enhancement in HHG originates from an intricate nonlinear coupling between the intraband and interband transitions that are respectively induced by perpendicular electric field components of the elliptically polarized light. Furthermore, we reveal that contributions from different excitation channels destructively interfere with each other. This finding suggests a path to potentially enhance the HHG by blocking a part of the channels and canceling the destructive interference through band-gap or chemical potential manipulation.
\end{abstract}

DOI: 10.1103/PhysRevB.103.L041408

High-order harmonic generation ( $\mathrm{HHG})$ is an extreme photon-upconversion process based on highly nonlinear lightmatter interactions. HHG was originally observed in atomic gas systems more than 30 years ago $[1,2]$. Several years later, the microscopic mechanism underlying $\mathrm{HHG}$ in noble gases was beautifully explained by a simple semiclassical model, the so-called three-step model [3-5]. On the practical side, high coherence in these upconversion processes allows us to generate extremely short light pulses, presenting a novel avenue to time-domain investigations of ultrafast electron dynamics in matter [6-14].

Since the first observation of HHG in solids by Gimire et al. [15], HHG in solids has been attracting much interest as it may have various applications ranging from the development of novel light sources [16] to probing of microscopic information of matter [17-19]. So far, experimental studies on HHG in solids have explored various materials [20-24], and the theoretical aspects of HHG in solids have been intensively investigated with various approaches [25-30].

In conventional nonlinear optics, the interplay of intraband and interband transitions plays an essential role [31,32]. Furthermore, recent attosecond spectroscopy clarified the significant roles of the nonlinear coupling among these transitions even in ultrafast and highly nonlinear optical phenomena

\footnotetext{
*ssato@ccs.tsukuba.ac.jp

†angel.rubio@mpsd.mpg.de
}

Published by the American Physical Society under the terms of the Creative Commons Attribution 4.0 International license. Further distribution of this work must maintain attribution to the author(s) and the published article's title, journal citation, and DOI. Open access publication funded by the Max Planck Society.
$[11,33,34]$. Hence the nonlinear coupling of intraband and interband transitions may be a key to accessing the microscopic physics behind light-induced phenomena. While intraband and interband transitions have been discussed in the context of HHG [25,35], the detailed roles of nonlinear coupling of these transitions still need to be investigated.

Yoshikawa et al. recently reported that the $\mathrm{HHG}$ in graphene can be enhanced by elliptically polarized light [23]. This observation is distinct from the HHG in noble gases, where HHG is significantly suppressed with an increase in the ellipticity of light [36-38]. Therefore, HHG in graphene under elliptically polarized light would offer an opportunity to look into the microscopic mechanism underlying HHG in solids. However, the mechanism of the enhancement is still unclear, although HHG in graphene has been intensively investigated [39-46].

In this Letter, we investigate the enhancement of HHG in graphene with elliptically polarized light, by employing a quantum master equation with a simple two-band model. The simple model of graphene fairly captures the experimentally observed enhancement of HHG with realistic conditions and provides a microscopic insight into the mechanism. The model indicates a significant role of the nonlinear coupling between light-induced intraband and interband transitions in the enhancement of HHG, demonstrating a destructive interference among multiple HHG channels.

To describe the electron dynamics, we employ a quantum master equation with a two-band approximation for the Dirac cone of graphene $[47,48]$. In the model, the time propagation of the one-body reduced density matrix at each Bloch wave vector $\boldsymbol{k}$ is described by

$$
\frac{d}{d t} \rho_{k}(t)=\frac{1}{i \hbar}\left[H_{k+A(t)}, \rho_{k}(t)\right]+\hat{D}\left[\rho_{k}(t)\right],
$$


where $H_{\boldsymbol{k}+\boldsymbol{A}(t)}$ is the Hamiltonian, and $\hat{D}\left[\rho_{\boldsymbol{k}}(t)\right]$ is a relaxation operator. In this Letter, we employ the following $2 \times 2$ Hamiltonian matrix,

$$
H_{k+A(t)}=v_{F} \tau_{z} \sigma_{x}\left[k_{x}+A_{x}(t)\right]+v_{F} \sigma_{y}\left[k_{y}+A_{y}(t)\right]+\frac{\Delta}{2} \sigma_{z},
$$

where $\sigma_{j}$ are Pauli matrices, $k_{j}$ are the $j$ components of the Bloch wave vector $\boldsymbol{k}$, and $A_{j}(t)$ is the $j$ component of the vector potential $\boldsymbol{A}(t)$, which corresponds to the applied electric fields, $\boldsymbol{E}(t)=-\dot{\boldsymbol{A}}(t)$. The band gap $\Delta$ is set to zero for graphene unless stated otherwise. Here, $\tau_{z}$ determines the chirality of the system (either +1 or -1 ). We evaluate observables as the average of two calculations with opposite chiralities. We set the Fermi velocity $v_{F}$ to $1.12 \times 10^{6} \mathrm{~m} / \mathrm{s}$ in accordance with an $a b$ initio simulation [49]. The relaxation operator $\hat{D}\left[\rho_{\boldsymbol{k}}(t)\right]$ is constructed by making the relaxationtime approximation [50] with a longitudinal relaxation time of $T_{1}=100 \mathrm{fs}$ and transverse relaxation time of $T_{2}=20 \mathrm{fs}$ $[47,48,51]$. Note that the relaxation operator also depends on the chemical potential $\mu ; \mu$ is set to zero (charge neutrality point) unless stated otherwise.

To describe the applied electric fields, we employ the following form of the vector potentials,

$$
\begin{aligned}
\boldsymbol{A}(t)= & -\frac{c E_{0, x}}{\omega_{0}} \boldsymbol{e}_{x} \cos \left(\omega_{0} t-\frac{\pi}{4}\right) \cos ^{4}\left(\frac{\pi}{T_{\text {full }}} t\right) \\
& -\frac{c E_{0, y}}{\omega_{0}} \boldsymbol{e}_{y} \cos \left(\omega_{0} t+\frac{\pi}{4}\right) \cos ^{4}\left(\frac{\pi}{T_{\text {full }}} t\right),
\end{aligned}
$$

in the domain $-T_{\text {full }} / 2<t<T_{\text {full }} / 2$; the potential is zero outside this domain. In accordance with the experimental conditions in Ref. [23], we set the mean photon energy $\hbar \omega_{0}$ to $260 \mathrm{meV}$. The full pulse duration $T_{\text {full }}$ is set to $100 \mathrm{fs}$. We will investigate the electron dynamics by changing the peak field strength of the applied laser fields $E_{0, x}$ and $E_{0, y}$.

Employing a time-dependent density matrix $\rho_{k}(t)$, we can evaluate the induced electric current as

$$
\boldsymbol{J}\left(t, E_{0, x}, E_{0, y}\right)=\frac{1}{(2 \pi)^{2}} \int d \boldsymbol{k} \operatorname{Tr}\left[\hat{\boldsymbol{J}}_{\boldsymbol{k}}(t) \rho_{\boldsymbol{k}}(t)\right],
$$

where $\hat{\boldsymbol{J}}_{\boldsymbol{k}}(t)$ is the current operator defined by

$$
\hat{\boldsymbol{J}}_{\boldsymbol{k}}(t)=-\frac{\partial H_{\boldsymbol{k}+\boldsymbol{A}(t)}}{\partial \boldsymbol{A}(t)} .
$$

Note that the current defined in Eq. (4) depends on $E_{0, x}$ and $E_{0, y}$ via $A(t)$ in Eq. (3), and for clarity we will indicate this dependence in the next equation by using the notation $\boldsymbol{J}\left(t, E_{0, x}, E_{0, y}\right)$.

In experiments, HHG occurs not only at the center of the beam spot but also on the whole focal area. To make our model more realistic, we employ the following intensity-averaging procedure to approximate the results for the case of a Gaussian beam profile $[28,51]$ :

$$
\boldsymbol{J}^{\mathrm{ave}}(t)=\int_{0}^{1} d \alpha \frac{1}{\alpha} \boldsymbol{J}\left(t, \alpha E_{0, x}, \alpha E_{0, y}\right) .
$$
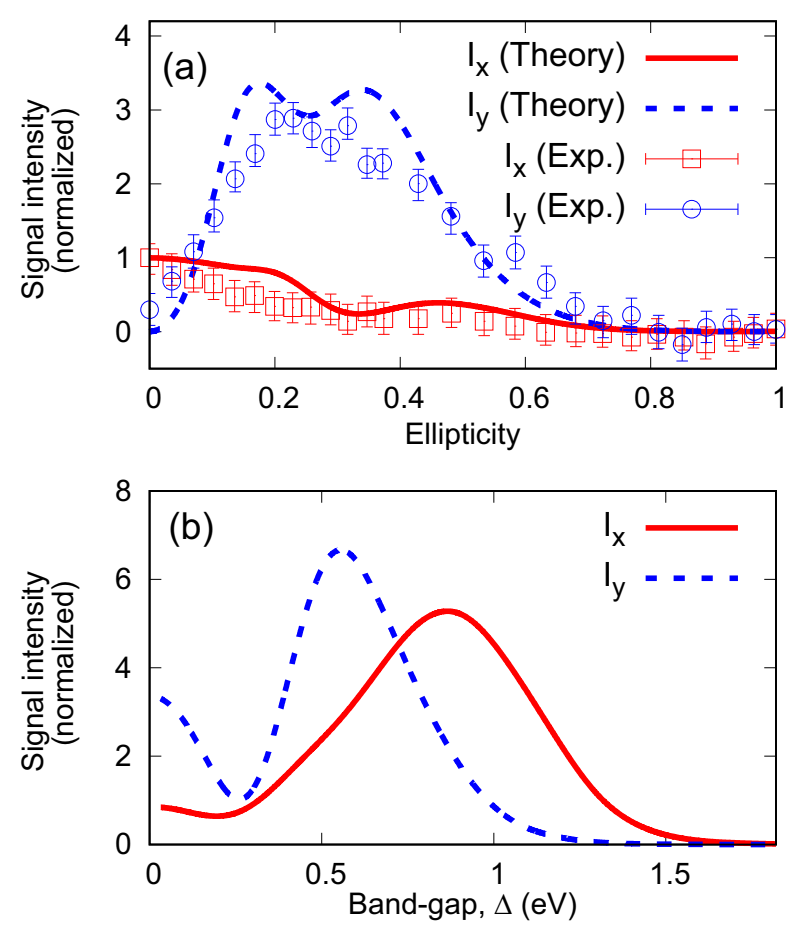

FIG. 1. The seventh-order harmonic intensity from graphene under elliptically polarized light: (a) Harmonic intensity as a function of ellipticity $E_{0, y} / E_{0, x}$. The experimental data [23] are also shown: (b) Harmonic intensity as a function of the band gap $\Delta$. The harmonic intensity is decomposed into the two polarization axes: the major $I_{x}^{7 \text { th }}$ (red solid) and minor $I_{y}^{7 \text { th }}$ (blue dotted) axes of the driving elliptically polarized light.

The power spectrum of the high-order harmonics polarized along the $j$ direction can be evaluated with the current as

$$
I_{j}(\omega) \sim \omega^{2}\left|\int d t J_{j}^{\text {ave }}(t) e^{i \omega t}\right|^{2},
$$

where $J_{j}^{\text {ave }}(t)$ is the $j$ component of the current vector $J^{\text {ave }}(t)$. Furthermore, the intensity of the $n$ th-order harmonics can be evaluated by integrating the power spectrum within a finite range as

$$
I_{j}^{n \text {th }}=\int_{n \omega_{0}-\frac{1}{2} \omega_{0}}^{n \omega_{0}+\frac{1}{2} \omega_{0}} d \omega I_{j}(\omega) .
$$

First, we evaluate the ellipticity dependence of the HHG by fixing the peak field strength $\sqrt{E_{0, x}^{2}+E_{0, y}^{2}}$ to $6.5 \mathrm{MV} / \mathrm{cm}$ inside the material. The major axis of the elliptically polarized light is set to the $x$ axis while the minor axis is set to the $y$ axis. Figure 1(a) shows the signal intensity of the seventh-order harmonics as a function of laser ellipticity $E_{0, y} / E_{0, x}$. The intensity $I_{j}^{\text {th }}$ is separately computed for the different polarization directions $(j=x$ or $j=y$ ) of the emitted harmonics. As seen from the figure, when the applied laser field is linearly polarized in the $x$ direction, the emitted high harmonics are also linearly polarized in the $x$ direction. Once the applied fields become elliptical, the emitted harmonics also become elliptical, having both $x$ and $y$ components. Interestingly, the $y$ component $I_{y}^{7 \text { th }}$ rapidly increases with the increase in driver 
ellipticity and becomes much larger than the $x$ component $I_{x}^{7 \text { th }}$, demonstrating the enhancement in HHG in graphene by elliptically polarized light. Once the driver ellipticity approaches one, the emitted harmonics is significantly suppressed due to the circular symmetry of the Dirac cone [52]. The fair agreement between the theory and experiment [23] demonstrates that the two-band model with the relaxation-time approximation contains sufficient ingredients to describe the HHG in graphene.

To obtain further insight into the phenomena, we evaluated the harmonic intensity by changing the band gap $\Delta$. Figure 1(b) shows the seventh-order harmonic intensity as a function of the band gap $\Delta$. Here, we used the same field strength as in Fig. 1(a). The ellipticity is set to 0.17 , and by the harmonic intensity is maximized at $\Delta=0$. Surprisingly, the harmonic intensity can be significantly enhanced by increasing the band gap. Furthermore, the $x$ component of the harmonic intensity $I_{x}^{\text {7th }}$ shows a peak around a band gap of $0.8 \mathrm{eV}$, which is close to the energy of three photons $(0.78 \mathrm{eV})$, while the $y$ component $I_{y}^{7 \text { th }}$ shows a peak around a band gap of $0.5 \mathrm{eV}$, which is close to the energy of two photons $(0.52 \mathrm{eV})$. The enhancement and formation of peaks that occur as the band gap increases indicate that multiphoton processes play a significant role in HHG in graphene, while Zener tunneling is expected to have only a minor contribution in the present regime.

The enhancement in HHG with the increase in band gap may be regarded as a counterintuitive consequence because an increase in the gap tends to block a part of the transitions. In previous works, the important role of the zero (or small) band gap of graphene has been suggested $[23,41]$. In fact, the high-order harmonics vanish once the band gap becomes significantly large, as shown in Fig. 1(b). To understand the enhancement in HHG with the increase in the gap, we propose a microscopic mechanism based on the destructive interference between multiple channels: High-order harmonics are generated as a superposition of multiple signals from various microscopic paths due to the nonlinear coupling of intraband and interband transitions. We further suppose that the multiple signals may destructively interfere with each other, and the total signal may be weakened. When such destructive interference plays a significant role, HHG may be enhanced by increasing the gap, because in so doing contributions can be partly suppressed, and the destructive interference can be canceled.

To examine our hypothesis, let us investigate the interference of different HHG contributions from the viewpoint of intraband and interband transitions. Here, we partly turn off the transitions based on the instantaneous eigenbasis representation [53,54], where the intraband transitions appear in the diagonal elements of the Hamiltonian and the interband transitions appear in the off-diagonal elements [51,55]. Elliptically polarized light consists of two polarization components, and each of them induces intraband and interband transitions. Hence, we can consider the four kinds of lightinduced transitions. For later convenience, we label them as follows: intraband transitions $\left(\tau_{a}\right)$ and interband transitions $\left(\tau_{b}\right)$ induced by the $x$ component of the electric fields; likewise, intraband transitions $\left(\tau_{c}\right)$ and interband transitions $\left(\tau_{d}\right)$ induced by the $y$ component. Figures 2(a)-2(d) show
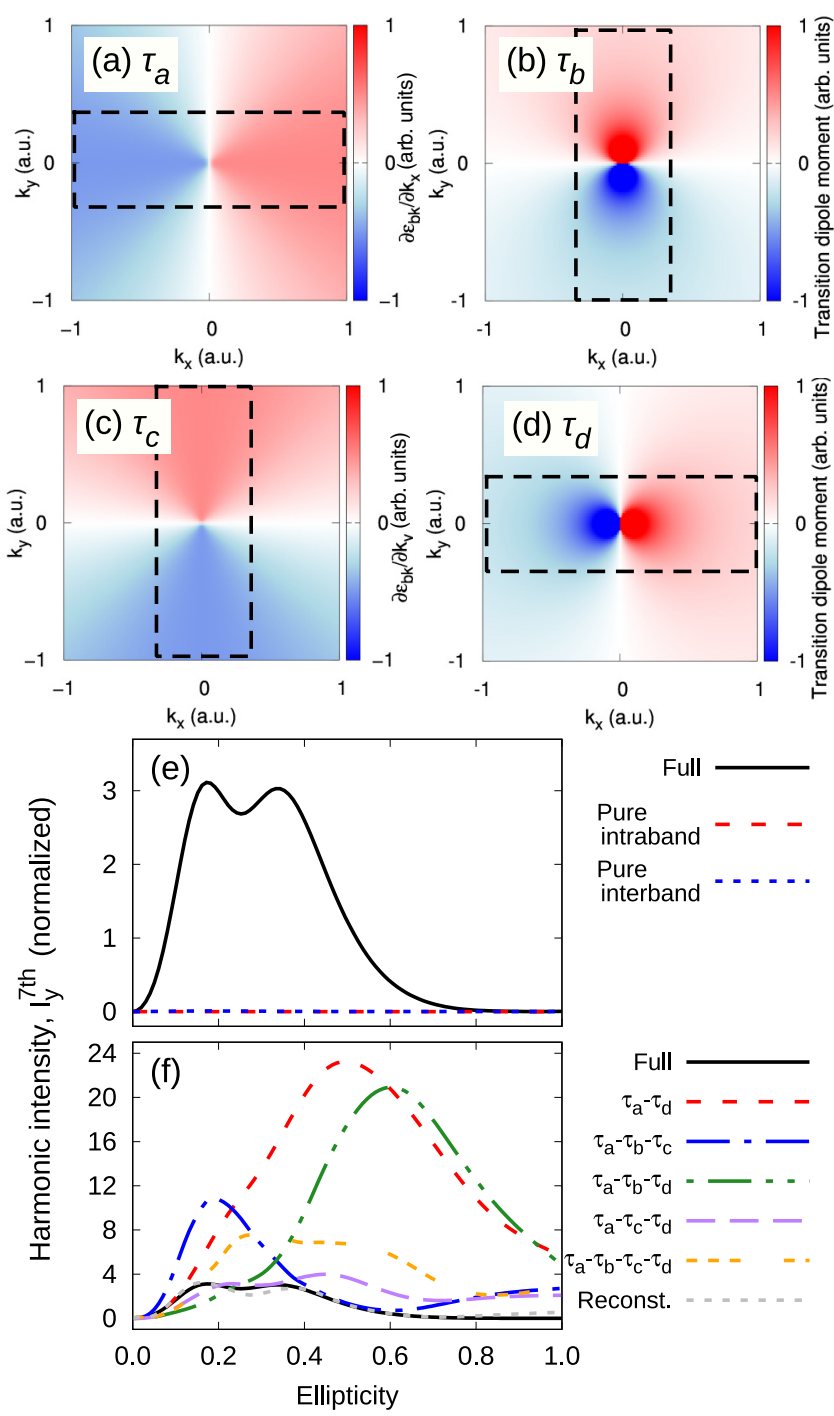

FIG. 2. (a)-(d) Strength profiles of intraband and interband transitions in $k$ space. The origin is set to the Dirac point. Strong interaction areas are indicated by black dashed squares. (e) The seventh-order harmonic intensity with the full intraband and interband transitions (black solid line), solely with the intraband transitions (red dashed line), and solely with the interband transitions (blue dotted line). (f) The seventh-order harmonic intensity with various decomposed transitions.

the strength distributions of each transition in $k$ space. Here, the strength of the intraband transitions is evaluated as the gradient of the single-particle energy $\partial \epsilon_{b, k} / \partial \boldsymbol{k}$, because the main contribution from the intraband transitions is the modulation of the dynamical phase factor $\exp \left[-i \int^{t} d t^{\prime} \epsilon_{b, \boldsymbol{k}+\boldsymbol{A}\left(t^{\prime}\right)}\right]$. The strength of the interband transitions is evaluated by the transition dipole moment. As seen from Figs. 2(a) and 2(b), the intraband and interband transitions induced by the $x$ component of the electric fields have alternating strength distributions in $k$ space; when one transition becomes stronger, the other becomes weaker. On the other hand, as seen from Figs. 2(a) and 2(d), the intraband and interband transitions induced respectively by the perpendicular components of the electric fields have a similar strength distribution. 
To elucidate the roles of the intraband and interband transitions in HHG, we can compute the electron dynamics by turning off part of them. In Fig. 2(e), the seventh-order harmonic intensity for the $y$ direction, $I_{y}^{7 \text { th }}$, with both intraband and interband transitions is shown as the black solid line. The results for only intraband transitions are shown as the red dashed line, while those for only interband transitions are shown as the blue dotted line. Here, we have used the same conditions as in Fig. 1(b) except the band gap $\Delta$, which is set to a small value of $0.035 \mathrm{eV}$ to avoid numerical singularity in the intraband-interband transition analysis at the Dirac point. As the figure clearly shows, neither pure intraband nor interband transitions can induce the HHG. Therefore, HHG originates from a nonlinear coupling of interband and interband transitions.

To study the coupling among intraband and interband transitions, we consider a decomposition of the current, extending the conventional decomposition in the nonlinear optics [31]. The current is decomposed into the coupling components of the transitions,

$$
\begin{aligned}
\boldsymbol{J}^{\mathrm{ave}}(t)= & \sum_{\tau} \boldsymbol{J}_{\tau}^{\mathrm{ave}}(t)+\sum_{\{\tau, \sigma\}} \boldsymbol{J}_{\tau, \sigma}^{\mathrm{ave}}(t) \\
& +\sum_{\{\tau, \sigma, \delta\}} \boldsymbol{J}_{\tau, \sigma, \delta}^{\mathrm{ave}}(t)+\boldsymbol{J}_{\tau_{a}, \tau_{b}, \tau_{c}, \tau_{d}}^{\mathrm{ave}}(t),
\end{aligned}
$$

where $\tau, \sigma$, and $\delta$ denote the labels of the transitions $\left(\tau_{a}, \tau_{b}\right.$, $\tau_{c}$, and $\tau_{d}$ ). In Eq. (9), there are 15 terms with four kinds of current: $\boldsymbol{J}_{\tau}^{\text {ave }}(t)$ is current induced solely by the transition $\tau$. $\boldsymbol{J}_{\tau, \sigma}^{\text {ave }}(t)$ is current induced by the coupling of two transitions, $\tau$ and $\sigma$. Likewise, $\boldsymbol{J}_{\tau, \sigma, \delta}^{\text {ave }}(t)$ is the current induced by the coupling among three transitions, $\tau, \sigma$, and $\delta$. Finally, $J_{\tau_{a}, \tau_{b}, \tau_{c}, \tau_{d}}^{\text {ave }}(t)$ is the current induced by the coupling of all four transitions. For more details, see Supplemental Material [51].

We evaluated the high-order harmonic intensity with the decomposed currents in Eq. (9) instead of the total current $J^{\text {ave }}(t)$. Figure 2(f) shows the seventh-order harmonic intensity $I_{y}^{7 \text { th }}$ as a function of ellipticity for various decomposed currents. Here, only the five major contributions, $\boldsymbol{J}_{\tau_{a}, \tau_{d}}^{\text {ave }}(t)$, $\boldsymbol{J}_{\tau_{a}, \tau_{b}, \tau_{c}}^{\mathrm{ave}}(t), \boldsymbol{J}_{\tau_{a}, \tau_{b}, \tau_{d}}^{\mathrm{ave}}(t), \boldsymbol{J}_{\tau_{a}, \tau_{c}, \tau_{d}}^{\mathrm{ave}}(t), \boldsymbol{J}_{\tau_{a}, \tau_{b}, \tau_{c}}^{\mathrm{ave}}(t)$, and $\boldsymbol{J}_{\tau_{a}, \tau_{b}, \tau_{c}, \tau_{d}}^{\mathrm{ave}}(t)$, are shown, while all the other contributions are rather minor [51]. In fact, the reconstructed signal from the five major contributions (gray dotted line) shows fair agreement with the full signal (black solid line). Remarkably, all the decomposed results in Fig. 2(b) have a larger harmonic intensity than the total signal. Hence the results clearly demonstrate destructive interference among the various contributions. Therefore, our hypothesis, i.e., destructive interference of HHG, is clearly supported by the theoretical results.

As seen from Fig. 2(f), the coupling of the intraband transition induced by the $x$ component of the electric fields $\left(\tau_{a}\right)$ and the interband transitions induced by the $y$ component of the fields $\left(\tau_{d}\right)$ shows the largest contribution to the harmonic intensity (red dashed line). Therefore, the cross-coupling between the intraband and interband transitions induced respectively by the perpendicular components of the electric fields plays a significant role in the enhancement of HHG in graphene under elliptically polarized light. In fact, all five major contributions include cross-coupling of the intraband and interband transitions with the perpendicular field compo-

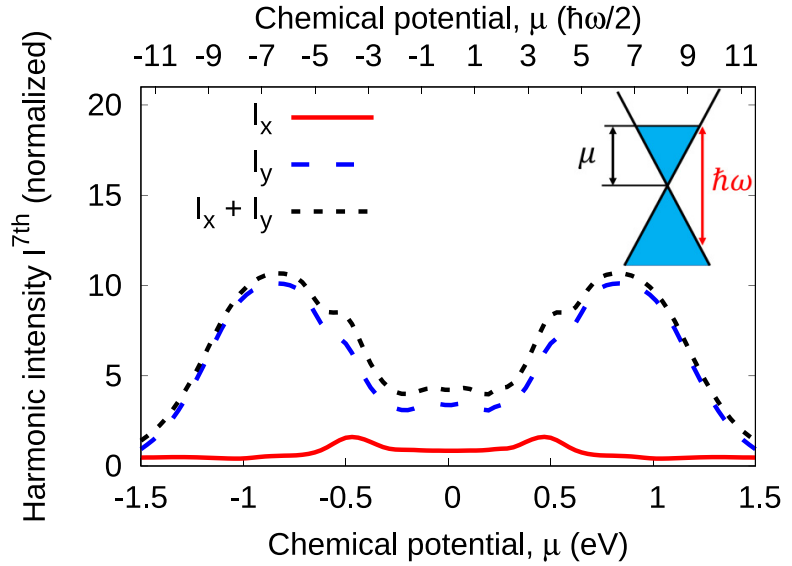

FIG. 3. The chemical potential dependence of the seventh-order harmonic intensity for a given ellipticity $E_{0, y} / E_{0, x}=0.17$.

nents. This observation can be straightforwardly understood in terms of the transition strength distribution in Figs. 2(a)2(d). Under linearly polarized light, the induced intraband and interband transitions have alternating strength distributions in $k$ space; when one transition becomes stronger, the other becomes weaker. Hence the coupling of the intraband and interband transitions is expected to be weak under linearly polarized light. In contrast, under elliptically polarized light, the intraband and interband transitions induced respectively by the perpendicular components of light have similar strength distributions; when one transition becomes stronger, the other also becomes stronger. Hence, the coupling of the intraband and interband transitions becomes stronger, resulting in an enhancement in HHG in graphene under elliptically polarized light.

Having established the destructive interference mechanism of HHG in solids, we propose a way to enhance HHG by canceling the destructive interference with the chemical potential shift. Since the chemical potential shift suppresses a part of the transitions in graphene by Pauli blocking (see the inset of Fig. 3), the destructive interference of multiple channels may be canceled by the tuning of the chemical potential, and this should result in an enhancement of HHG. To demonstrate the impact of the chemical potential shift, we evaluate the seventh-order harmonic intensity from graphene by changing the chemical potential $\mu$. Figure 3 shows the seventh-order harmonic intensity as a function of the chemical potential $\mu$. Here, we used the same field strength as Fig. 1(b) and set the ellipticity to 0.17 . As shown in Fig. 3, the high-order harmonic intensity can be enhanced by tuning the chemical potential. Hence the proposed method of enhancement in the HHG based on the destructive interference mechanism has clearly been demonstrated. Furthermore, the total harmonic intensity shows the peak around $|\mu| \sim 1 \mathrm{eV} \sim 7 \hbar \omega / 2$. Note that, once the absolute value of the chemical potential reaches half of the $n$ th-photon energy, $|\mu| \sim n \hbar \omega_{0} / 2$, the $n$-photon resonant processes are suppressed by the Pauli blocking. Thus, the enhancement in HHG and the peak feature in Fig. 3 further indicate the significance of multiphoton processes in the present regime. 
In conclusion, we investigated and identified the microscopic mechanism underlying HHG in graphene exposed to elliptically polarized light by employing the quantum master equation with the simple Dirac cone [47,51]. We found that the nonlinear coupling between the intraband and interband transitions is the microscopic origin of the HHG in graphene. In particular, the cross-coupling between the intraband and interband transitions induced by the perpendicular components of the electric fields causes the enhancement in HHG under elliptically polarized light, reflecting the unique transition strength profiles of graphene in $k$ space, as shown in Figs. 2(a)-2(d). Our findings of the interference effects on HHG will lead to a general understanding of HHG in solids, and also provide novel techniques to increase the HHG efficiency. Indeed, we have demonstrated that the high-order harmonics can be enhanced by tuning the chemical potential $\mu$ or the band gap $\Delta$, by blocking a part of the transitions.
Furthermore, the interference mechanism may open a way to control the high-order harmonic generation by tuning phases of each channel contribution and by flipping the destructive interference to constructive interference through the optimization of applied laser fields such as the relative phase control of a two-color laser.

This work was supported by JSPS KAKENHI Grants No. JP20K14382 and No. JP19H05465, the European Research Council (ERC-2015-AdG694097), the International Collaborative Research Program of Institute for Chemical Research, Kyoto University (Grant No. 2020-13), the Cluster of Excellence "Advanced Imaging of Matter" (AIM), Grupos Consolidados (IT1249-19), and SFB925 "Light induced dynamics and control of correlated quantum systems." The Flatiron Institute is a division of the Simons Foundation.
[1] A. McPherson, G. Gibson, H. Jara, U. Johann, T. S. Luk, I. A. McIntyre, K. Boyer, and C. K. Rhodes, J. Opt. Soc. Am. B 4, 595 (1987).

[2] M. Ferray, A. L'Huillier, X. F. Li, L. A. Lompre, G. Mainfray, and C. Manus, J. Phys. B: At., Mol. Opt. Phys. 21, L31 (1988).

[3] K. Kulander, K. Schafer, and J. Krause, Super-Intense LaserAtom Physics (Springer, Berlin, 1993), pp. 95-110.

[4] P. B. Corkum, Phys. Rev. Lett. 71, 1994 (1993).

[5] M. Lewenstein, P. Balcou, M. Y. Ivanov, A. L'Huillier, and P. B. Corkum, Phys. Rev. A 49, 2117 (1994).

[6] T. Brabec and F. Krausz, Rev. Mod. Phys. 72, 545 (2000).

[7] F. Krausz and M. Ivanov, Rev. Mod. Phys. 81, 163 (2009).

[8] E. Goulielmakis, Z.-H. Loh, A. Wirth, R. Santra, N. Rohringer, V. S. Yakovlev, S. Zherebtsov, T. Pfeifer, A. M. Azzeer, M. F. Kling, S. R. Leone, and F. Krausz, Nature (London) 466, 739 (2010).

[9] A. R. Beck, B. Bernhardt, E. R. Warrick, M. Wu, S. Chen, M. B. Gaarde, K. J. Schafer, D. M. Neumark, and S. R. Leone, New J. Phys. 16, 113016 (2014).

[10] M. Schultze, K. Ramasesha, C. Pemmaraju, S. Sato, D. Whitmore, A. Gandman, J. S. Prell, L. J. Borja, D. Prendergast, K. Yabana, D. M. Neumark, and S. R. Leone, Science 346, 1348 (2014).

[11] M. Lucchini, S. A. Sato, A. Ludwig, J. Herrmann, M. Volkov, L. Kasmi, Y. Shinohara, K. Yabana, L. Gallmann, and U. Keller, Science 353, 916 (2016).

[12] M. Zürch, H.-T. Chang, L. J. Borja, P. M. Kraus, S. K. Cushing, A. Gandman, C. J. Kaplan, M. H. Oh, J. S. Prell, D. Prendergast, C. D. Pemmaraju, D. M. Neumark, and S. R. Leone, Nat. Commun. 8, 15734 (2017).

[13] F. Siegrist, J. A. Gessner, M. Ossiander, C. Denker, Y.-P. Chang, M. C. Schröder, A. Guggenmos, Y. Cui, J. Walowski, U. Martens, J. K. Dewhurst, U. Kleineberg, M. Münzenberg, S. Sharma, and M. Schultze, Nature (London) 571, 240 (2019).

[14] M. Volkov, S. A. Sato, F. Schlaepfer, L. Kasmi, N. Hartmann, M. Lucchini, L. Gallmann, A. Rubio, and U. Keller, Nat. Phys. 15, 1145 (2019).

[15] S. Ghimire, A. D. DiChiara, E. Sistrunk, P. Agostini, L. F. DiMauro, and D. A. Reis, Nat. Phys. 7, 138 (2011).
[16] S. Ghimire and D. A. Reis, Nat. Phys. 15, 10 (2019).

[17] G. Vampa, T. J. Hammond, N. Thiré, B. E. Schmidt, F. Légaré, C. R. McDonald, T. Brabec, D. D. Klug, and P. B. Corkum, Phys. Rev. Lett. 115, 193603 (2015).

[18] A. Chacón, W. Zhu, S. P. Kelly, A. Dauphin, E. Pisanty, A. Picón, C. Ticknor, M. F. Ciappina, A. Saxena, and M. Lewenstein, Phys. Rev. B 102, 134115 (2020).

[19] R. E. F. Silva, Á. Jiménez-Galán, B. Amorim, O. Smirnova, and M. Ivanov, Nat. Photonics 13, 849 (2019).

[20] O. Schubert, M. Hohenleutner, F. Langer, B. Urbanek, C. Lange, U. Huttner, D. Golde, T. Meier, M. Kira, S. W. Koch, and R. Huber, Nat. Photonics 8, 119 (2014).

[21] T. T. Luu, M. Garg, S. Y. Kruchinin, A. Moulet, M. T. Hassan, and E. Goulielmakis, Nature (London) 521, 498 (2015).

[22] Y. S. You, D. A. Reis, and S. Ghimire, Nat. Phys. 13, 345 (2017).

[23] N. Yoshikawa, T. Tamaya, and K. Tanaka, Science 356, 736 (2017).

[24] Y. Sanari, H. Hirori, T. Aharen, H. Tahara, Y. Shinohara, K. L. Ishikawa, T. Otobe, P. Xia, N. Ishii, J. Itatani, S. A. Sato, and Y. Kanemitsu, Phys. Rev. B 102, 041125(R) (2020).

[25] D. Golde, T. Meier, and S. W. Koch, Phys. Rev. B 77, 075330 (2008).

[26] G. Vampa, C. R. McDonald, G. Orlando, D. D. Klug, P. B. Corkum, and T. Brabec, Phys. Rev. Lett. 113, 073901 (2014).

[27] G. Vampa, C. R. McDonald, G. Orlando, P. B. Corkum, and T. Brabec, Phys. Rev. B 91, 064302 (2015).

[28] I. Floss, C. Lemell, G. Wachter, V. Smejkal, S. A. Sato, X.-M. Tong, K. Yabana, and J. Burgdörfer, Phys. Rev. A 97, 011401(R) (2018).

[29] T. Ikemachi, Y. Shinohara, T. Sato, J. Yumoto, M. KuwataGonokami, and K. L. Ishikawa, Phys. Rev. A 95, 043416 (2017).

[30] N. Tancogne-Dejean, O. D. Mücke, F. X. Kärtner, and A. Rubio, Phys. Rev. Lett. 118, 087403 (2017).

[31] C. Aversa and J. E. Sipe, Phys. Rev. B 52, 14636 (1995).

[32] J. E. Sipe and A. I. Shkrebtii, Phys. Rev. B 61, 5337 (2000). 
[33] F. Schlaepfer, M. Lucchini, S. A. Sato, M. Volkov, L. Kasmi, N. Hartmann, A. Rubio, L. Gallmann, and U. Keller, Nat. Phys. 14, 560 (2018).

[34] B. Buades, A. Picón, I. León, N. Di Palo, S. L. Cousin, C. Cocchi, E. Pellegrin, J. H. Martin, S. Mañas-Valero, E. Coronado et al., arXiv:1808.06493.

[35] D. Golde, T. Meier, and S. W. Koch, Phys. Status Solidi C 6, 420 (2009).

[36] K. S. Budil, P. Salières, A. L'Huillier, T. Ditmire, and M. D. Perry, Phys. Rev. A 48, R3437 (1993).

[37] P. Dietrich, N. H. Burnett, M. Ivanov, and P. B. Corkum, Phys. Rev. A 50, R3585(R) (1994).

[38] Y. Liang, M. V. Ammosov, and S. L. Chin, J. Phys. B: At., Mol. Opt. Phys. 27, 1269 (1994).

[39] A. K. Gupta, O. E. Alon, and N. Moiseyev, Phys. Rev. B 68, 205101 (2003).

[40] S. A. Mikhailov, Europhys. Lett. 79, 27002 (2007).

[41] K. L. Ishikawa, Phys. Rev. B 82, 201402(R) (2010).

[42] I. Al-Naib, J. E. Sipe, and M. M. Dignam, Phys. Rev. B 90, 245423 (2014).

[43] Ó. Zurrón, A. Picón, and L. Plaja, New J. Phys. 20, 053033 (2018).

[44] H. A. Hafez, S. Kovalev, J.-C. Deinert, Z. Mics, B. Green, N. Awari, M. Chen, S. Germanskiy, U. Lehnert, J. Teichert, Z. Wang, K.-J. Tielrooij, Z. Liu, Z. Chen, A. Narita, K. Müllen,
M. Bonn, M. Gensch, and D. Turchinovich, Nature (London) 561, 507 (2018).

[45] P. Bowlan, E. Martinez-Moreno, K. Reimann, T. Elsaesser, and M. Woerner, Phys. Rev. B 89, 041408(R) (2014).

[46] M. Taucer, T. J. Hammond, P. B. Corkum, G. Vampa, C. Couture, N. Thiré, B. E. Schmidt, F. Légaré, H. Selvi, N. Unsuree, B. Hamilton, T. J. Echtermeyer, and M. A. Denecke, Phys. Rev. B 96, 195420 (2017).

[47] S. A. Sato, J. W. McIver, M. Nuske, P. Tang, G. Jotzu, B. Schulte, H. Hübener, U. De Giovannini, L. Mathey, M. A. Sentef, A. Cavalleri, and A. Rubio, Phys. Rev. B 99, 214302 (2019).

[48] S. A. Sato, P. Tang, M. A. Sentef, U. D. Giovannini, H. Hübener, and A. Rubio, New J. Phys. 21, 093005 (2019).

[49] P. E. Trevisanutto, C. Giorgetti, L. Reining, M. Ladisa, and V. Olevano, Phys. Rev. Lett. 101, 226405 (2008).

[50] T. Meier, G. von Plessen, P. Thomas, and S. W. Koch, Phys. Rev. Lett. 73, 902 (1994).

[51] See Supplemental Material at http://link.aps.org/supplemental/ 10.1103/PhysRevB.103.L041408 for more details.

[52] O. E. Alon, V. Averbukh, and N. Moiseyev, Phys. Rev. Lett. 80, 3743 (1998).

[53] W. V. Houston, Phys. Rev. 57, 184 (1940).

[54] J. B. Krieger and G. J. Iafrate, Phys. Rev. B 33, 5494 (1986).

[55] S. A. Sato, M. Lucchini, M. Volkov, F. Schlaepfer, L. Gallmann, U. Keller, and A. Rubio, Phys. Rev. B 98, 035202 (2018). 